\title{
Intrinsically Passive Force Scaling in Haptic Interfaces
}

\author{
Cristian Secchi ${ }^{1}$, Stefano Stramigioli ${ }^{2}$, Cesare Fantuzzi ${ }^{1}$ \\ ${ }^{1}$ DISMI, Univ. of Modena and Reggio Emilia \\ Viale Allegri 13, 42100 Reggio Emilia, Italy \\ \{secchi.cristian,fantuzzi.cesare\}@unimore.it \\ ${ }^{2}$ EEMCS, University of Twente \\ P.O.Box 217, 7500 AE Enschede, NL \\ S.Stramigioli@ieee.org
}

\begin{abstract}
In several applications involving haptic interfaces it can be desirable to scale the interaction force perceived by the user. The most intuitive approach is to change the stiffness of the virtual environment but, unfortunately, changing the physical parameters that characterize a virtual environment is a potentially destabilizing action. In this paper we embed in the intrinsically passive haptic scheme recently proposed in [1] a power scaling interconnection that allows to scale the force perceived by the user while preserving the passivity, and consequently the stable behavior, of the overall system.
\end{abstract}

\section{INTRODUCTION}

Haptic interfaces allow a user to interact with a simulated virtual environment by means of a robotic device. These interfaces have been exploited in many application domains as for example for practicing surgical procedure on virtual patients, for virtual training and for virtually designing and testing mechanical parts.

Haptic interfaces can be also used to assist the user in the execution of a given task. Virtual fixtures, introduced in [2], are perceptual overlays that help the human operator to execute a task with the required degree of precision. In other words, a virtual fixture is a simulated constraint that guides the user over a preferred path enhancing its tracking performance. They have been used for increasing the precision and/or the speed in path following and in positioning tasks [3], for training [4] and for improving performances in telemanipulation tasks [2], [5]. Virtual fixtures can be interpreted as virtual environments, characterized by a certain stiffness, with which the user is interacting during the execution of a task and which influence the operator's natural behavior by assisting him/her. Nevertheless, as pointed out in [6], by constraining the user's motion, a virtual fixture also limits the user's control over a given task. The authors point out that, because of an error in the design phase, it is possible that the fixture is misplaced and that therefore it may have a negative effect on the task execution. Furthermore, it may happen that there are certain regions where the user wants to defy the virtual fixture in order either to avoid an area or to reach a position that is not on the path marked by the fixture. It is then necessary to be able to scale the force imposed by the virtual constraint on the user in order to increase the level of user's control over the task and in order to allow him/her to reach off-fixture targets. In [6] impedance fixtures are considered and the force scaling issue is addressed by changing the stiffness of the virtual constraint; several algorithms for varying the stiffness are considered and compared experimentally.

An haptic interface is an interactive robotic system and, therefore, stability is a key issue since both oscillations and unstable behaviors can lead to unnatural feedback from the virtual environment. Passivity has been widely used as a guideline for designing haptic interfaces characterized by a stable behavior and several control strategies for guaranteeing the intrinsic passivity of an haptic interface have been proposed in the literature as for example the virtual coupling [7] and the PO/PC strategy [8].

When using an intrinsically passive haptic interface any non passive operation during the execution of the task should be avoided in order to preserve a stable behavior. Thus, while a virtual fixture characterized by a certain stiffness can be passively implemented, changing the stiffness of a virtual environment is a non-passive operation. In fact, consider for example a 1-DOF linear spring with stiffness $k>0$ and kept at a constant elongation $x>0$. The energy of the spring in this configuration is given by $H_{1}=1 / 2 k x^{2}$ and if we change the stiffness to a value $k_{1}>k$ the energy stored is now given by $H_{2}=1 / 2 k_{1} x^{2}>H_{1}$ and, therefore, by changing the stiffness we introduced an extra energy amount equal to $H_{2}-H_{1}$. This injection of extra energy can give rise to regenerative effects that can make the interaction with the virtual environment unstable. For further details and the treatment of the general case see [9].

In [6], it is reported that the users have some difficulties in using the system when they have to go back to the virtual constraint from the off fixture target, namely when the stiffness of the virtual environment they are interacting with increases; we think that this is related to the regenerative, non passive, effect associated to the stiffness increase.

An haptic interface can be modeled as the energetic interconnection of four systems as shown in Fig. 1 where bond-graph notation has been used (the double band on the bond indicates that the "virtual" energetic exchange is in the discrete domain). Both the human operator and the haptic device are passive systems while some extra energy injection can derive both from the Sample \& Hold procedure ( $S H$ in Fig. 1) and from 


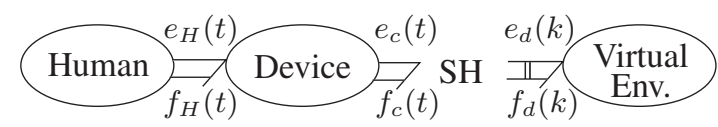

Fig. 1. Energetic representation of a haptic display

the simulation of the discrete virtual environment.

In [1] a new approach for the implementation of an haptic interface has been proposed. The authors have implemented in a passive way each possible source of energy. First, a Sample \& Hold algorithm which preserves passivity has been introduced. By means of this algorithm, it is possible to connect a continuous and a discrete system without the production of any extra energy. In order to be able to represent any passive physical system, the port-Hamiltonian formalism [10] has been adopted to represent virtual environments and a passivity preserving discretization algorithm has been introduced. Having implemented any possible source of extra energy in a passive way, it has been possible to obtain a passive haptic interface by interconnecting in a power preserving way [10] each component represented in Fig. 1.

The goal of this paper is to replace the $S H$ strategy proposed in [1], that allows a lossless energy transfer between the haptic device and the virtual environment, with the power scaled interconnection proposed in [11]. We will prove that in this way it is possible to scale both the velocity imposed by the user and the force transmitted by the virtual environment without affecting the passivity of the overall scheme. In fact, in this way force scaling doesn't derive from an alteration of the physical properties of the virtual environment but from the interposition of a tunable layer, whose behavior doesn't affect the passivity of the overall system, between the haptic device and the virtual environment. Using the proposed strategy it is possible to scale the feeling perceived by the user while preserving a stable behavior of the system; in particular, it is possible to scale the constraining effect of a virtual fixture in a safe, intrinsically stable way.

The paper is organized as follows: in Sec. II we will give some background on port-Hamiltonian systems and on the scheme proposed in [1] and in Sec. III we will introduce the power scaled interconnection and we will prove that it is possible to passively scale the interaction with the virtual environment. In Sec. IV we will show that, under suitable conditions, it is possible to implement a time-varying scaling while preserving the passivity of the overall scheme. In Sec. V we will provide some simulations in order to validate the results obtained in the paper and in Sec. VI some conclusions are reported and future work is addressed.

\section{BACKGROUND}

\section{A. Port-Hamiltonian systems}

We can consider a port-Hamiltonian system as composed of a state manifold $\mathcal{X}$, an energy function $H: \mathcal{X} \rightarrow \Re$ corresponding to the internal energy, a network structure $D(x)=-D(x)^{T}$ whose graph has the mathematical structure of a Dirac structure([10]), which is in general a state dependent power continuous interconnection structure, and an interconnection port (power port) represented by a pair of dual variables $(e, f) \in V^{*} \times V$ called effort and flow respectively. This port is used to interact energetically with the system. The power supplied through a port is equal to $e(f)$ or using coordinates to $e^{T} f$. We can furthermore split the interaction port in more sub-ports, each of which can be used to model different power flows. We will indicate with the subscript $I$ the power ports by means of which the system interacts with the rest of the world, with the subscript $C$ the power ports associated with the storage of energy and with the subscript $R$ the power ports relative to the dissipative part. Summarizing, we have:

$$
\left(\begin{array}{c}
e_{I} \\
f_{C} \\
e_{R}
\end{array}\right)=D(x)\left(\begin{array}{c}
f_{I} \\
e_{C} \\
f_{R}
\end{array}\right)
$$

A dissipating element of the system can be modeled using as characteristic equations $e_{R}=R(x) f_{R}$ with $R(x)$ a symmetric and positive semi-definite matrix. If we furthermore set $\dot{x}=$ $f_{C}$ and $e_{C}=\frac{\partial H}{\partial x}$, due to the previous power balance we obtain:

$$
\dot{H}+f_{R}^{T} R(x) f_{R}=e_{I}^{T} f_{I}
$$

which clearly says that the supplied power $e_{I}^{T} f_{I}$ equals the increase of internal energy $H$ plus the dissipated one and that, therefore, a port-Hamiltonian system is passive. For further details the reader is addressed to [10]. A very broad class of physical system can be modeled as a port-Hamiltonian system and therefore this formalism can be successfully used to describe both linear and nonlinear environments. In the development of haptic interfaces is necessary to find a discrete time representation of a physical system which has to be simulated as virtual environment. Unfortunately, passivity is not preserved using standard discretization techniques and, therefore, it can happen that the discrete counterpart of a passive system becomes active and, therefore, characterized by an unstable behavior.

A port-Hamiltonian system can be passively discretized using the algorithm proposed in [1]. In this case the following discrete power balance holds:

$$
\Delta H(k)+T f_{R}^{T}(k) R(x(k)) f_{R}(k)=T e_{I}^{T}(k) f_{I}(k)
$$

where $H(x(k))$ is the discrete energy function, $T$ is the sample period, $\left(e_{R}(k), f_{R}(k)\right)$ and $\left(e_{I}(k), f_{I}(k)\right)$ are the discrete power ports associated to the dissipation and to the interaction with the rest of the world respectively and $\Delta H(k)=H(x(k+$ $1))-H(x(k))$. For further details the reader is referred to [1].

\section{B. Port-Hamiltonian Haptics}

In order to let the user to interact with the virtual environment it is necessary to couple a continuous haptic device with a discrete port-Hamiltonian system describing a virtual environment. Using standard sample and hold techniques, some regenerative, destabilizing effects, can be introduced in the haptic interface. In [1] a simple algorithm to guarantee a 


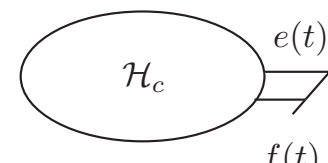

SH

$f(t)$

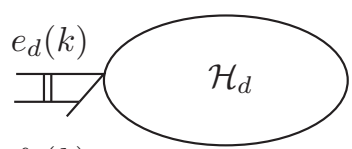

$f_{d}(k)$
Fig. 2. The interconnection of discrete and continuous port-Hamiltonian systems

lossless exchange of energy between a physical device and a virtual environment has been introduced.

Consider the port interconnection of a continuous time Hamiltonian system $H_{C}$ and a discrete Hamiltonian system $H_{D}$ through a sampler and zero-order hold as shown in Fig. 2. Suppose that $H_{C}$ has an admittance causality (effort in/flow out) and that, therefore, $H_{D}$ has an impedance causality (flow in/effort out). We will have that:

$$
e(t)=e_{d}(k) \quad t \in[k T,(k+1) T]
$$

The following theorem can be proven [1]:

Theorem 1 (Sampled Data Passivity): If we define for the interconnection port of $H_{D}$

$$
f_{d}(k):=\frac{q(k T)-q((k+1) T)}{T},
$$

where $q(\cdot)$ represents the integral of the continuous flow, we obtain an equivalence between the continuous time and discrete time energy flow in the sense that for each $n$ :

$$
\sum_{i=0}^{n-1} e_{d}^{T}(i) f_{d}(i)=-\int_{0}^{n T} e^{T}(s) f(s) d s
$$

From the previous considerations, it is possible to understand that at each sampling time, we have an EXACT matching between the physical energy going into the continuous time system and the virtual energy coming from the discrete time port. Notice that in haptic interfaces the variable $q(\cdot)$ corresponds to the position of the haptic device.

Thus, a generic haptic interface can be modeled as the passive interconnection of a continuous port-Hamiltonian system, representing the haptic device, and of passive discrete portHamiltonian system. The overall system is passive and therefore characterized by a stable behavior.

\section{Power SCALING IN PORT-HAMILTONIAN BASED HAPTIC INTERFACES}

In this section we consider port-Hamiltonian based haptic interfaces and we will prove that it is possible to scale the feeling of interaction with the virtual environment perceived by the human operator while preserving the passivity, and, therefore, the stable behavior, of the overall system. We will use the following notation for the discrete derivative and the discrete integral:

$$
d g(k)=\frac{g(k+1)-g(k)}{T} \quad I_{h}^{k} g=\sum_{i=h}^{k-1} g(i) T
$$

where $g$ is a generic sequence.
Since the haptic device and the virtual environment are passive systems the following energy balances hold:

$$
\begin{aligned}
& \int_{0}^{n T}\left(e_{H}^{T}(\tau) f_{H}(\tau)+e_{c}^{T}(\tau) f_{c}(\tau)\right) d \tau= \\
& =H_{c}\left(x_{c}(n T)\right)-H_{c}\left(x_{c}(0)\right)+E_{\text {diss }}^{c}(n T)
\end{aligned}
$$

and

$$
I_{0}^{n} e_{d}^{T}(k) f_{d}(k)=H_{d}\left(x_{d}(n T)\right)-H_{d}\left(x_{d}(0)\right)+E_{d i s s}^{d}(n T)
$$

where $x_{c}$ and $x_{d}$ are the states of the haptic device and of the virtual environment respectively, $H_{c}$ and $H_{d}$ are lower bounded functions representing the energy stored in the continuous haptic device and in the virtual environment and $E_{\text {diss }}^{c}$ and $E_{\text {diss }}^{d}$ are nonnegative functions representing the energy dissipated by the systems; we assumed that $E_{\text {diss }}^{c}(0)=0$ and that $E_{\text {diss }}^{d}(0)=0$, namely that at the beginning the haptic device is at rest and that the virtual environment is at equilibrium. Furthermore, when using the interconnection strategy reported in Sec. II-B we have that the following balance holds:

$$
I_{0}^{n} e_{d}^{T}(i) f_{d}(i)=-\int_{0}^{n T} e_{c}^{T}(\tau) f_{c}(\tau) d \tau \quad \forall n \in \mathbb{N}
$$

which says that at any sampling instant the energy extracted by the continuous haptic device is supplied to the virtual environment and viceversa and,therefore, that no extra energy is produced in the interconnection. Scaling the interaction means to scale the exchange of energy between the haptic device and the virtual environment which, on its turn, means scaling the effort and the flow exchanged between the device and the virtual environment. Thus, we propose to use the following power scaled sample and hold strategy:

$$
\left\{\begin{array}{l}
f_{d}(k)=-\alpha \frac{q(k+1)-q(k)}{T} \\
e_{c}(t)=\frac{1}{\beta} \operatorname{hold}\left(e_{d}(k)\right)
\end{array} \quad \alpha, \beta \in \mathbb{R}^{+}\right.
$$

where $\alpha$ and $\beta$ are factors that allow to scale the interaction of the user with the environment. If we are interested in scaling only the force transmitted to the user (as in the case of virtual fixtures studied in [6]) we can set $\alpha=1$ and tune $\beta$ for achieving the desired force scaling. Using this interconnection, we have that

$$
-\frac{1}{\alpha \beta} I_{0}^{n} e_{d}^{T}(i) f_{d}(i)=\int_{0}^{n T} f_{c}^{T}(\tau) e_{c}(\tau) d \tau \forall n \in \mathbb{N}
$$

which means that the energy extracted by the virtual environment is scaled and supplied to the haptic device. Thus, the exchange of energy with the virtual environment is perceived by the user scaled by a factor $1 / \alpha \beta$. Thus the interconnection strategy reported in Eq.(7) is not passivity preserving because, in general, the power extracted from one side can be amplified and supplied to the other side, leading to a production of energy. Nevertheless, the following result can be proven:

Proposition 1: If the haptic device is a passive system, if the virtual environment is rendered as a discrete passive system and if the interconnection between the continuous and the 
discrete part is made through the Sample and Hold strategy reported in Eq.(7), then the overall system is passive.

Proof. Since the haptic device and the virtual environment are passive systems, both Eq.(4) and Eq.(5) hold and, consequently, for each sampling instant we have that:

$$
\begin{aligned}
& \alpha \beta \int_{0}^{n T} e_{H}^{T}(\tau) f_{H}(\tau) d \tau+\alpha \beta \int_{0}^{n T} e_{c}^{T}(\tau) f_{c}(\tau) d \tau+ \\
& +I_{0}^{n} e_{d}^{T}(i) f_{d}(i)=\alpha \beta\left[H _ { c } \left(x_{c}(n T)-H_{c}\left(x_{c}(0)\right]+\right.\right. \\
& +H_{d}\left(x_{d}(n T)\right)-H_{d}\left(x_{d}(0)+\alpha \beta E_{d i s s}^{c}(n T)+\right. \\
& +E_{d i s s}^{d}(n T)
\end{aligned}
$$

Since the interconnection between the haptic device and the virtual environment is made through Eq.(7) we have that, using Eq.(8):

$$
I_{0}^{n} e_{d}^{T}(i) f_{d}(i)=-\alpha \beta \int_{0}^{n T} e_{c}^{T}(\tau) f_{c}(\tau) d \tau
$$

Using Eq.(10) in Eq.(9) and taking into account that $\alpha \beta E_{\text {diss }}^{c}(\cdot)$ and $E_{\text {diss }}^{d}(\cdot)$ are nonnegative functions, it follows that:

$$
\begin{aligned}
& \alpha \beta \int_{0}^{n T} e_{H}^{T}(\tau) f_{H}(\tau) d \tau \geq \alpha \beta\left[H _ { c } \left(x_{c}(n T)-H_{c}\left(x_{c}(0)\right]+\right.\right. \\
& +H_{d}\left(x_{d}(n T)\right)-H_{d}\left(x_{d}(0)\right.
\end{aligned}
$$

whence

$$
\begin{aligned}
& \int_{0}^{n T} e_{H}^{T}(\tau) f_{H}(\tau) d \tau \geq H_{c}\left(x_{c}(n T)-H_{c}\left(x_{c}(0)+\right.\right. \\
& +\frac{1}{\alpha \beta}\left[H_{d}\left(x_{d}(n T)\right)-H_{d}\left(x_{d}(0)\right]\right.
\end{aligned}
$$

which proves that the system is passive with respect to the storage function

$$
H_{c}(\cdot)+\frac{1}{\alpha \beta} H_{d}(\cdot)
$$

The scaled interconnection is NOT a passive element but, nevertheless, it can be safely used in the implementation of an haptic interface. This happens because the effect of the scaling is to "mask" the virtual environment and to make it appear to the human operator as if it acted at a different power scale transmitting, in particular, to him/her a scaled force. However, this amplification/attenuation of the power transmitted does NOT modify the kind of dynamic behavior of the virtual environment which keeps on being passive. Changing the stiffness of the virtual environment, instead, deeply influence the kind of dynamic behavior of the virtual environment and the passivity of the overall haptic interface cannot be guaranteed anymore.

\section{VARIABLE SCALING}

An abrupt change in the perception of the virtual environment can disturb the user and therefore, as suggested in [6] for virtual fixtures, a gradual attenuation of the scaling has to be preferred. Thus, it is necessary to consider variable factors that gradually scale the interaction with the virtual environment.
In other words, we should use the following variable power scaled sample and hold interconnection:

$$
\left\{\begin{array}{l}
f_{d}(k)=-\alpha(k) \frac{q(k+1)-q(k)}{T} \\
e c(t)=\frac{1}{\beta(k)} \operatorname{hold}\left(e_{d}(k)\right)
\end{array}\right.
$$

where $\alpha(k)$ and $\beta(k)$ are the bounded scaling factors and

$$
\begin{aligned}
& \underline{\alpha} \leq \alpha(k) \leq \bar{\alpha} \quad \underline{\alpha}, \bar{\alpha} \in \mathbb{R}^{+} \\
& \underline{\beta} \leq \beta(k) \leq \bar{\beta} \quad \underline{\beta}, \bar{\beta} \in \mathbb{R}^{+}
\end{aligned}
$$

In this case, the effect of the power scaling interconnection is no more static and, therefore, the variation of $\alpha$ and $\beta$ can introduce some dynamic effects which could destroy the intrinsic passivity of the haptic interface. Luckily, if some not very restrictive conditions on the kind of virtual environment are satisfied, we can still preserve the passivity of the overall scheme.

The following lemma will be useful in the following:

Lemma 1: Let $f: \mathbb{R} \mapsto \mathbb{R}$ be a real function such that

$$
\int_{0}^{t} \dot{f}(\tau) d \tau \geq-\delta \quad \forall t \quad \delta \in \mathbb{R}^{+}, \delta<\infty
$$

and let $\gamma: \mathbb{R} \mapsto \mathbb{R}^{+}$be a scaling function such that

$$
\underline{\gamma} \leq \gamma(t) \leq \bar{\gamma} \quad \forall t \quad \underline{\gamma}, \bar{\gamma} \in \mathbb{R}^{+}
$$

Suppose that the function $f(\cdot)$ has a finite number of critical points, namely that its derivative changes sign a finite number of times. Then

$$
\int_{0}^{t} \gamma(\tau) \dot{f}(\tau) d \tau
$$

is lower bounded.

Proof. Let $\operatorname{sign}(\cdot)$ indicate the sign function. Consider an interval $\left[t_{0}, t_{1}\right]$ such that $\operatorname{sign}(\dot{f}(t))=$ const. $\forall t \in\left[t_{0}, t_{1}\right]$. We can distinguish three cases. If $\operatorname{sign}(\dot{f}(t))=1$ then

$$
\begin{aligned}
& \int_{t_{0}}^{t_{1}} \gamma(\tau) \dot{f}(\tau) d \tau \geq \underline{\gamma}\left(f\left(t_{1}\right)-f\left(t_{0}\right)\right)= \\
& \underline{\gamma}\left(f\left(t_{1}\right)-f(0)\right)+\underline{\gamma}\left(f(0)-f\left(t_{0}\right)\right) \geq \\
& \geq-\underline{\gamma} \delta+\underline{\gamma}\left(f(0)-f\left(t_{0}\right)\right)
\end{aligned}
$$

If $\operatorname{sign}(\dot{f}(t))=0$ then

$$
\int_{t_{0}}^{t_{1}} \gamma(\tau) \dot{f}(\tau) d \tau=0
$$

If $\operatorname{sign}(\dot{f}(t))=-1$ then

$$
\begin{aligned}
& \int_{t_{0}}^{t_{1}} \gamma(\tau) \dot{f}(\tau) d \tau \geq \bar{\gamma}\left(f\left(t_{1}\right)-f(0)\right)+ \\
& +\bar{\gamma}\left(f(0)-f\left(t_{0}\right)\right) \geq-\bar{\gamma} \delta+\bar{\gamma}\left(f(0)-f\left(t_{0}\right)\right)
\end{aligned}
$$

Every interval $[0, t]$ can be split up in the following way

$$
[0, t]=\left[0, t_{1}\right] \bigcup \cup_{i=1}^{p} I_{p_{i}} \bigcup \cup_{j=1}^{n} I_{n_{j}} \bigcup \cup_{k=1}^{z} I_{z_{k}}
$$


where

$$
\begin{aligned}
& I_{p_{i}}=\left[t_{i}, \bar{t}_{i}\right] \quad \bar{t}_{i}>t_{i} \quad \operatorname{sign}(\dot{f}(t))=1 \forall t \in I_{p_{i}} \\
& I_{n_{j}}=\left[t_{j}, \bar{t}_{j}\right] \quad \bar{t}_{j}>t_{j} \quad \operatorname{sign}(\dot{f}(t))=-1 \forall t \in I_{n_{j}} \\
& I_{z_{k}}=\left[t_{k}, \bar{t}_{k}\right] \quad \bar{t}_{k}>t_{k} \quad \operatorname{sign}(\dot{f}(t))=0 \forall t \in I_{n_{k}}
\end{aligned}
$$

and where $\operatorname{sign}(\dot{f}(t))=$ const. $\forall t \in\left[0, t_{1}\right]$. Let $\Gamma$ be defined as:

$$
\Gamma= \begin{cases}\bar{\gamma} & \text { if } \operatorname{sign}(\dot{f}(t))=-1 \forall t \in\left[0, t_{1}\right] \\ 0 & \text { if } \operatorname{sign}(\dot{f}(t))=1 \forall t \in\left[0, t_{1}\right] \\ \underline{\gamma} & \text { if } \operatorname{sign}(\dot{f}(t))=1 \forall t \in\left[0, t_{1}\right]\end{cases}
$$

Thus we can write

$$
\begin{aligned}
& \int_{0}^{t} \gamma(\tau) \dot{f}(\tau) d \tau \geq \Gamma \int_{0}^{t_{1}} \dot{f}(\tau) d \tau+ \\
& \sum_{i=0}^{p} \int_{t_{i}}^{\bar{t}_{i}} \gamma(\tau) \dot{f}(\tau) d \tau+\sum_{j=1}^{n} \int_{t_{j}}^{\bar{t}_{j}} \gamma(\tau) \dot{f}(\tau) d \tau \geq-\Gamma \delta+ \\
& +\sum_{i=0}^{p}\left(-\underline{\gamma} \delta+\underline{\gamma}\left(f(0)-f\left(t_{i}\right)\right)\right)+ \\
& +\sum_{j=0}^{n}\left(-\bar{\gamma} \delta+\bar{\gamma}\left(f(0)-f\left(t_{j}\right)\right)\right)=-\Gamma \delta-p \underline{\gamma} \delta- \\
& -n \bar{\gamma} \delta+\underline{\gamma} \sum_{i=0}^{p}\left(f(0)-f\left(t_{i}\right)\right)+\bar{\gamma} \sum_{j=0}^{n}\left(f(0)-f\left(t_{j}\right)\right)
\end{aligned}
$$

$f\left(t_{i}\right)$ and $f\left(t_{j}\right)$ are finite since they are critical points. Furthermore, because of the hypothesis, $p<\infty$ and $n<\infty$ and therefore the sums are finite and consequently the integral is lower bounded.

Thanks to the lemma it is possible to build interfaces where the haptic flow can be variably scaled. In fact we can prove the following result:

Proposition 2: Consider the scheme reported in Fig. 1 and suppose that the Sample \& Hold strategy is implemented through the strategy reported in Eq.(13). Suppose that the energy function that characterizes the virtual environment has a finite number of critical points. Then the overall haptic interface is passive.

Proof. Set $\alpha(k) \beta(k)=\gamma(k), \underline{\alpha} \beta=\underline{\gamma}$ and $\bar{\alpha} \bar{\beta}=\bar{\gamma}$. We have that

$$
\gamma(i) \int_{i T}^{(i+1) T} e_{c}^{T}(\tau) f_{c}(\tau) d \tau=-e_{d}^{T}(i) f_{d}(i) T
$$

Furthermore we have that

$$
\begin{aligned}
& \int_{i T}^{(i+1) T} \gamma(i) e_{H}^{T}(\tau) f_{H}(\tau) d \tau+\int_{i T}^{(i+1) T} \gamma(i) e_{c}^{T}(\tau) f_{c}(\tau) d \tau+ \\
& +e_{d}^{T}(i) f_{d}(i) T=\gamma(i)\left[\int_{i T}^{(i+1) T} e_{H}^{T}(\tau) f_{H}(\tau) d \tau+\right. \\
& \left.+\int_{i T}^{(i+1) T} e_{c}^{T}(\tau) f_{c}(\tau) d \tau\right]+e_{d}^{T}(i) f_{d}(i) T= \\
& \gamma(i)\left(\Delta H_{c}(i)\right)+\Delta H_{d}(i)+\gamma(i) E_{\text {diss }}^{c}(i)+E_{\text {diss }}^{d}(i)
\end{aligned}
$$

where $\Delta H_{c}(i)=H_{c}\left(x_{c}((i+1) T)\right)-H_{c}\left(x_{c}(i T)\right)$ and $\Delta H_{d}(i)=H_{d}\left(x_{d}((i+1) T)\right)-H_{d}\left(x_{d}(i T)\right)$. Using Eq.(25) in Eq.(26) we have that:

$$
\int_{i T}^{(i+1) T} e_{H}^{T}(\tau) f_{H}(\tau) d \tau \geq \Delta H_{c}(i)+\frac{1}{\gamma(i)}\left(\Delta H_{d}(i)\right)
$$

Summing over $i=0,1, \ldots, n-1$ we have that

$$
\begin{aligned}
& \int_{0}^{n T} e_{H}^{T}(\tau) f_{H}(\tau) d \tau \geq H_{c}\left(x_{c}(n T)-H_{c}\left(x_{c}(0)+\right.\right. \\
& \sum_{i=0}^{n-1} \underbrace{\frac{1}{\gamma(i)} \Delta H_{d}(i)}_{\Delta \bar{H}_{d}}=H_{c}\left(x_{c}(n T)-H_{c}\left(x_{c}(0)+\right.\right. \\
& +\bar{H}_{d}(n T)-\bar{H}_{d}(0)
\end{aligned}
$$

$H_{c}$ is a lower bounded function and, since $H_{d}(\cdot)$ has a finite number of critical points and since $\gamma(i)$ is a bounded positive function, a straightforward application of the discrete version of Lemma 1 allows to conclude that also $\bar{H}_{d}(\cdot)$ is lower bounded. Thus, the energy balance reported in Eq.(28) proves that the overall system is passive.

Remark 1: The assumptions made by the lemma are not very restrictive and they are satisfied by the majority of energy functions associated to the virtual environments that are simulated in haptic interfaces. Nevertheless, they are necessary. In fact, we can passively simulate a physical environment characterized by an energy function $H(x)=\sin (x)$ but in case of variable scaling, this virtual environment would lead to an unstable interaction.

If the conditions reported in Proposition 2 are satisfied, it is possible to use the interconnection reported in Eq.(13) for changing the perception of the virtual environment at each instant while preserving passivity, and consequently a stable behavior, of the overall system.

\section{Simulations}

The aim of this section is to provide some simulations in order to validate the results obtained in the paper.

We consider a 1-DOF intrinsically passive haptic interface. The haptic device is a simple mass of $1 \mathrm{Kg}$ characterized by some friction that is modeled as a viscous friction with coefficient $b=0.1 \mathrm{Ns} / \mathrm{m}$. The virtual environment is the discrete equivalent of a spring, that is obtained by the passivity preserving discretization algorithm described in Sec. II-A and it is joined to the haptic device through the interconnection strategy reported in Eq.(13). The overall system is passive and, therefore, it is characterized by a stable behavior.

We suppose that the virtual environment plays the role of a very simple $1-\mathrm{DOF}$ virtual fixture that constrains the user at $x=0 \mathrm{~m}$ and that the stiffness of the virtual fixture has been set to a small value $(10 \mathrm{~N} / \mathrm{m})$ to allow the user to easily reach off-fixture configurations. In order to take back the user to the fixture position, it is necessary to scale the force transmitted to the user. In order to show its effectiveness, we will compare the scaling strategy proposed in the paper with the effect that would be obtained by directly changing the stiffness of the 
virtual environment. Thus, suppose that the the user is keeping the haptic device at the position $x_{0}=1 \mathrm{~m}$ applying the relatively small force $F=10 \mathrm{~N}$. At time $t=10 \mathrm{~s}$, the user has to be taken back to the fixture position $(x=0 \mathrm{~m})$ and, therefore, the force applied from the virtual environment has to be scaled.

In order to test the effect of the change of stiffness, we don't introduce any scaling between the haptic device and the virtual environment and, therefore, we use the lossless interconnection described in Sec. II-B.

In Fig. 3 the stiffness is changed linearly from $10 \mathrm{~N} / \mathrm{m}$ to $400 \mathrm{~N} / \mathrm{m}$, corresponding to scaling the force perceived by the user of a factor 40; the overall behavior is unstable. The instability is related to the variation of the stiffness, which introduces energy into the system and which consequently breaks the passivity.

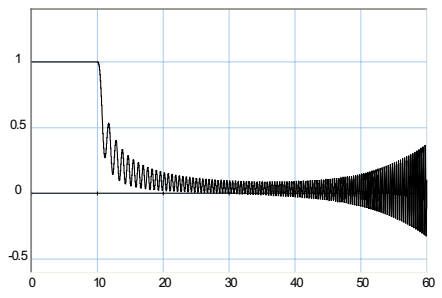

(a) Position

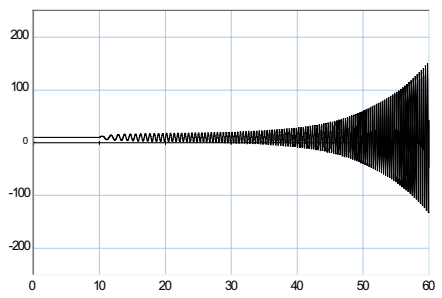

(b) The force exchanged with the user
Fig. 3. A gradual change of stiffness

In the next simulation we don't change the stiffness of the virtual fixture but we scale the force transmitted to the human using the strategy proposed in this paper. Since we are interested in scaling only the force, we set $\alpha=1$ and we let $\beta$ changing linearly from the value 1 to the value 1000 . The results of the simulation are shown in Fig. 4.

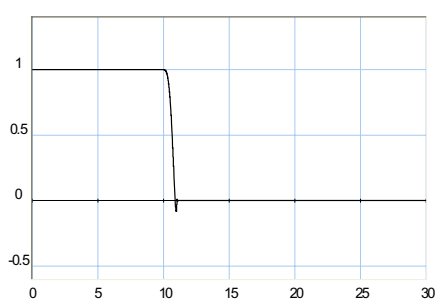

(a) Position

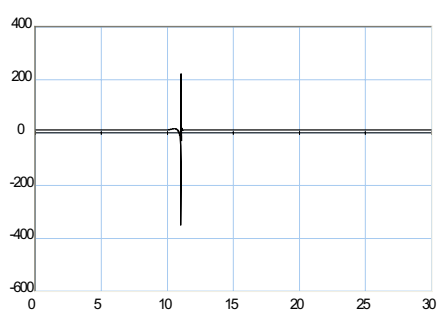

(b) The force exchanged with the user
Fig. 4. The proposed scaling strategy

We can see that, even if the force scaling factor is much bigger than that implemented with the stiffness variation, the behavior of the overall system is stable. This happens because the force scaling is achieved without violating the passivity and the conditions of Proposition 2 are satisfied.

\section{CONCLUSIONS AND FUTURE WORK}

In this paper we have considered the port-Hamiltonian based intrinsically passive haptic control scheme and we have shown how it is possible to embed both velocity and force scaling while preserving the passivity, and therefore a stable behavior, of the overall system.

The key feature of the intrinsic safety of our approach relies in recognizing that changing the physical characteristics of the virtual environment modifies drastically the dynamic behavior of the system and it can lead to a regenerative effect that destroys the passivity and that can lead to unstable behaviors. Using the scaled interconnection proposed in the paper, we don't change the kind of dynamic behavior of the virtual environment but we simply "mask" the perception transmitted to the user; this guarantees that the passivity of the overall system is preserved.

Future work aims at experimentally validating the results of the paper and at extending the scaling strategy to delayed virtual environment. As proven in [1], power variables cannot be used to interconnect the haptic device to the virtual environment since the delay would cause a loss of passivity. It is necessary to use scattering variables. Thus, the scaled interconnection reported in Eq.(13) does not guarantee the passivity anymore and, therefore, the scaling should be reformulated using scattering variables.

\section{Acknowledgments}

This work has been done in the context of the European sponsored project GeoPlex with reference code IST-200134166. Further information are available at www.geoplex. CC.

\section{REFERENCES}

[1] S. Stramigioli, C. Secchi, A. van der Schaft, and C. Fantuzzi, "Sampled data systems passivity and discrete port-hamiltonian systems," IEEE Transactions on Robotics, vol. 21, no. 4, pp. 574-587, 2005.

[2] 1. Rosenberg, "Virtual fixtures: Perceptual tools for telerobotic manipulation," in Proceedings of the Virtual Reality Annual International Symposium, 1993.

[3] A. Bettini, P. Marayong, S. Lang, A. Okamura, and G. HAger, "Visionassisted control for manipulation using virtual fixtures," IEEE Transactions on Robotics, vol. 20, pp. 953-966, 2004.

[4] S. Payandeh and Z. Stanisic, "On application of virtual fixtures as an aid for telemanipulation and training," in Proceedings of the Symposium on Haptic Interfaces for Virtual Environment and Teleoperator Systems, 2002.

[5] J. Abbot and A. Okamura, "Analysis of virtual fixture contact stability for telemanipulation," in Proceedings of IEEE/RSJ International Conference on Intelligent Robots and Systems, Las Vegas, NV, USA, 2003.

[6] J. Nolin, P. Stemniski, and A. Okamura, "Activation cues and force scaling methods for virtual fixtures," in Proceedings of the 11th Symposium on Haptic Interfaces for Virtual Environment and Teleoperator Systems, Chicago, IL, USA, 2003.

[7] J. Colgate and G. Schenkel, "Passivity of a class of sampled-data systems: Application to haptic interfaces," in Proceedings to American Control Conference, 1994.

[8] B. Hannaford and J.-H. Ryu, "Time domain passivity control of haptic interfaces," IEEE Transactions on Robotics and Automation, vol. 18, no. 1, pp. 1-10, February 2002.

[9] C. Secchi, "Interactive robotic interfaces: a port-hamiltonian approach," Ph.D. dissertation, University of Modena and Reggio Emilia, 2004, available at http://www.dismi.unimore.it/download/thesis.pdf.

[10] A. van der Schaft, $L_{2}$-Gain and Passivity Techniques in Nonlinear Control, ser. Communication and Control Engineering. Springer Verlag, 2000.

[11] C. Secchi, S. Stramigioli, and C. Fantuzzi, "Power scaling in port-hamiltonian based bilateral telemanipulation," in Proceedings of IEEE/RSJ International Conference on Intelligent Robots and Systems, Edmonton, Canada, August 2005. 